\title{
A population-based predictive model predicting candidate for primary tumor surgery in patients with metastatic esophageal cancer
}

\author{
Zhichao Liu"^, Xiaobin Zhang", Bin Li”, Haoyao Jiang, Yang Yang, Rong Hua, Yifeng Sun, Zhigang Li \\ Department of Thoracic Surgery, Section of Esophageal Surgery, Shanghai Chest Hospital, Shanghai Jiao Tong University, Shanghai, China \\ Contributions: (I) Conception and design: Z Liu, Z Li; (II) Administrative support: Z Li; (III) Provision of study materials or patients: All authors; (IV) \\ Collection and assembly of data: Z Liu, H Jiang, Y Yang, R Hua, Y Sun; (V) Data analysis and interpretation: Z Liu, X Zhang, B Li, H Jiang; (VI) \\ Manuscript writing: All authors; (VII) Final approval of manuscript: All authors. \\ \#These authors contributed equally to this work. \\ Correspondence to: Zhigang Li. Department of Thoracic Surgery, Section of Esophageal Surgery, Shanghai Chest Hospital, Shanghai Jiao Tong \\ University, 241 Huaihai West Rd, Shanghai 200030, China. Email: zhigang_li_sch@163.com.
}

Background: The survival benefit of primary tumor surgery for metastatic esophageal cancer (mEC) patients has been observed, but methods for discriminating which individual patients would benefit from surgery have been poorly defined. Herein, a predictive model was developed to test the hypothesis that only certain metastatic patients would gain a survival benefit from primary tumor surgery.

Methods: Clinical data for patients with mEC were extracted from the Surveillance, Epidemiology and End Results (SEER) database [2004-2016] and then divided into surgery and no-surgery groups according to whether surgery was performed on the primary tumor. Propensity-score-matching (PSM) was performed to balance the confounding factors. We hypothesized that the patients who had undergone surgery and lived longer than the median cancer-specific-survival (CSS) of the no-surgery group could benefit from surgery. We constructed a nomogram to predict surgery benefit potential based on multivariable logistic-regression analysis using preoperative factors. The predictive performance of the nomogram was evaluated by the area under the receiver operating characteristic (AUC) and calibration curves. The clinical application value of the nomogram was estimated with decision curve analysis (DCA).

Results: A total of 5,250 eligible patients with mEC were identified, and 9.4\% [492] received primary tumor surgery. After PSM, CSS for the surgery group was significantly longer [median: 19 vs. 9 months; hazard ratio $(\mathrm{HR})$ 0.52, $\mathrm{P}<0.001]$ compared with the no-surgery group. Among the surgery group, 69.3\% [327] survived $>9$ months (surgery-beneficial group). The prediction nomogram showed good discrimination both in training and validation sets (AUC: 0.72 and 0.70, respectively), and the calibration curves indicated a good consistency. DCA demonstrated that the nomogram was clinically useful. According to this nomogram, surgery patients were classified into two groups: no-benefit-candidate and benefit-candidate. The benefitcandidate group was associated with longer survival than the no-benefit-candidate group (median CSS: 19 vs. 6.5 months, $\mathrm{P}<0.001$ ). Additionally, there was no difference in survival between the no-benefit-candidate and no-surgery groups (median CSS: 6.5 vs. 9 months, $\mathrm{P}=0.070$ ).

Conclusions: A predictive model was created for the selection of candidates for surgical treatment among mEC patients. This predictive model might be used to select patients who may benefit from primary tumor surgery.

Keywords: Esophageal cancer (EC); metastatic; surgery; predictive model

Submitted Jul 01, 2020. Accepted for publication Dec 10, 2020.

doi: $10.21037 /$ jtd-20-2347

View this article at: http://dx.doi.org/10.21037/jtd-20-2347

\footnotetext{
$\wedge$ ORCID: 0000-0003-3542-0905.
} 


\section{Introduction}

Esophageal cancer (EC) is the seventh most common cancer worldwide and is the sixth leading cause of cancer-related death (1). Remarkably, metastatic esophageal cancer (mEC) accounts for approximately $40 \%$ of EC (2). Additionally, distant metastases eventually develop in $25 \%$ of patients with only locoregional disease at their initial diagnosis (3). Patients with metastatic stage disease are generally assumed to be incurable; treatment options are limited, and life expectancy is greatly reduced.

Systemic chemotherapy is considered the principal therapeutic strategy for $\mathrm{mEC}$ patients; however, this application of chemotherapy is largely palliative, there is limited evidence of durable benefit, and the median overall survival is approximately 10 months (4,5). Primary tumor surgery is not usually a recommended treatment for patients with mEC. The primary focus and goals of surgery in such patients are disease control and palliation without curative intent. Recent studies have suggested that surgery to the primary site is associated with improved survival for some mEC patients (6-8). However, only certain patient subpopulations with $\mathrm{mEC}$ would benefit from resection of the primary tumor due to the existence of substantial heterogeneity in clinicopathological characteristics among such patients (8-10). Given the lack of effective treatment options for mEC patients, surgery to control the primary tumor should be explored as an approach to improve the dismal outcomes of $\mathrm{mEC}$. The potential benefit of surgery to the primary tumor in $\mathrm{mEC}$ might vary among patients based on individual characteristics. The judicious selection of patients for such surgery is advisable; however, the current understanding of which patients would benefit the most is inadequate. Therefore, an individualized prediction model would help assist the selection for this situation.

Herein, this study aimed to develop a predictive model using a prospective national database to identify good candidates for primary tumor surgery in $\mathrm{mEC}$ patients. We present the following article in accordance with the TRIPOD reporting checklist (available at http://dx.doi. org/10.21037/jtd-20-2347).

\section{Methods}

\section{Patient and data sources}

The Surveillance, Epidemiology, and End Results (SEER) database is a national population-based registry program that collects tumor-related clinical data and basic demographics, covering approximately $28 \%$ of the US population.

The study was conducted in accordance with the Declaration of Helsinki (as revised in 2013). This study was based on the publicly available data from the SEER database, and we were granted permission to access the research data. As all the data were anonymous, it was deemed exempt by the Institutional Review Board of the Shanghai Chest Hospital.

Patients diagnosed with EC (tumor location coded as C15.0-C15.5 and C15.8-15.9) were identified during a study period of 2004 to 2016 from the SEER database using the SEER*Stat software (version 8.3.5) according to the site code classifications. We selected this range because the American Joint Committee on Cancer (AJCC) tumor node metastasis (TMN) stage and Collaborative Stage (CS) information were available from 2004 onwards. The TNM stage was reclassified according to the AJCC 8th edition to generate a uniform dataset. Additional inclusion criteria were as follows (Figure 1): (I) patients diagnosed as a metastatic stage with pathologically confirmed squamous cell carcinoma or adenocarcinoma; (II) with one primary tumor only. The exclusion criteria included patients with missing or incomplete (unknown) data such as TNM stage, survival status and time, or treatment information.

The baseline information and clinicopathological data of all eligible cases were collected and retrospectively analyzed. Surgery was defined as cancer-direct surgery on the primary site, including partial esophagectomy, total esophagectomy, local tumor excision, and esophagectomy with laryngectomy and/or gastrectomy. Cancer specific survival (CSS) was calculated from the date of diagnosis to the date of death attributed to EC.

\section{Statistical analysis}

According to primary tumor surgical treatment, the study population was divided into two groups: a surgery and a nosurgery group. To minimize selection bias and imbalanced distributions of the confounding factors, the propensity-score matching (PSM) was calculated to account for confounding variables and facilitate patient matching in the two treatment groups. Variables that could potentially influence treatment outcomes were used to generate a propensity score by logistic regression, including age, race, gender, tumor location, differentiation grade, histology, TNM stage, radiotherapy, chemotherapy, and surgery to distant site. Participants in the two groups (surgery and no-surgery) 


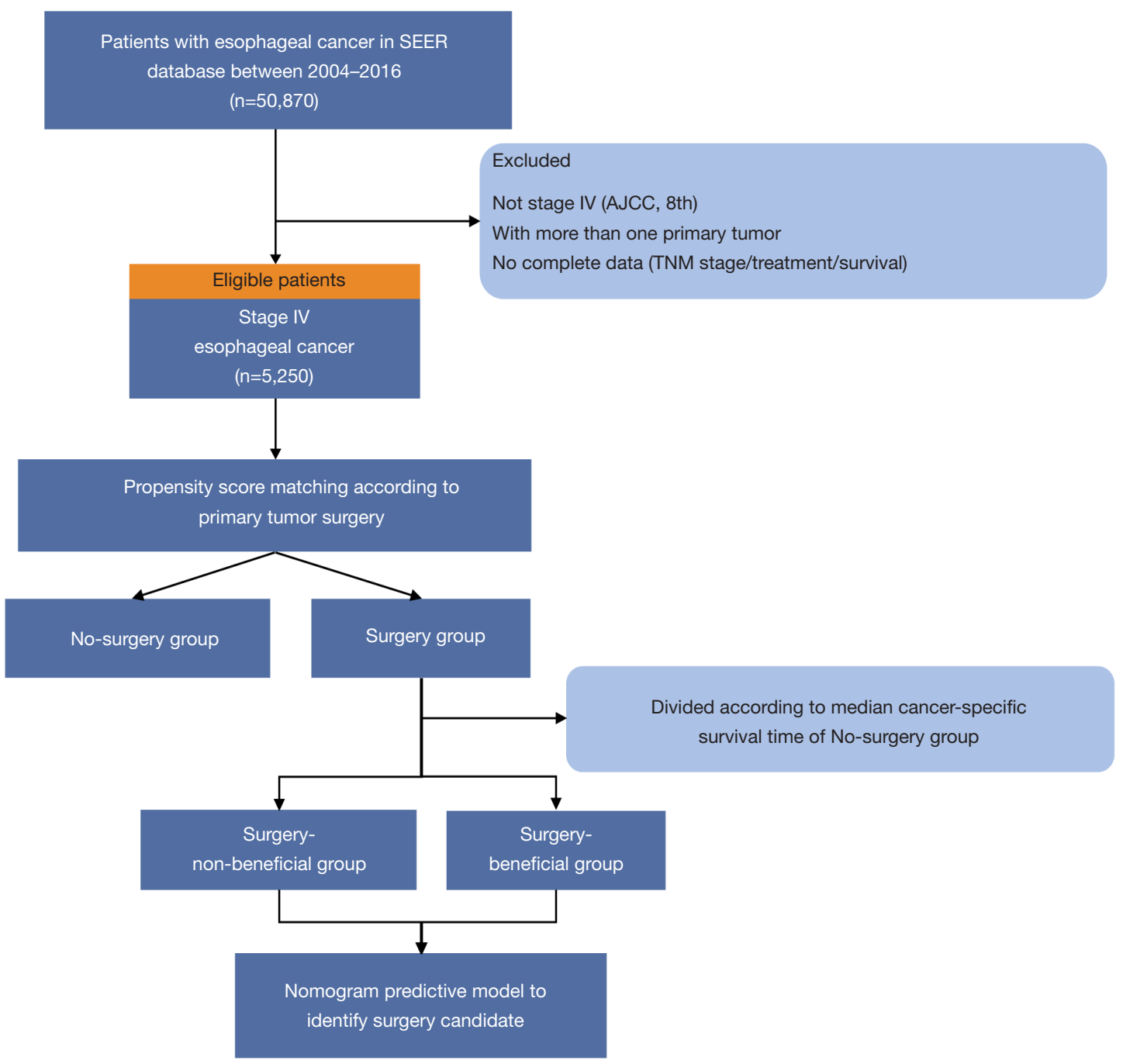

Figure 1 The flowchart of study population selection and predictive model construction.

were 1:1 matched using the nearest propensity score on the logit scale with a caliper of 0.1 without replacement (11). The balance of co-variables in both groups before and after PSM was evaluated by the standardized differences, and a value of standardized difference $<10 \%$ was considered as criteria of sufficient balance (12).

The distribution of variables across treatment groups was evaluated using the chi-square test for categorical variables and $t$-test for continuous variables. The CSS were estimated by the Kaplan-Meier method and compared with the logrank test. Cox proportional hazard regression was used to determine independent prognostic factors. Hazard ratio (HR) was calculated with $95 \%$ confidence intervals (CIs). Statistical analysis was performed with the software SPSS
25.0 (IBM Corporation, Armonk, NY, USA) and R version 3.6.3 software (http://www.r-project.org/). A two-sided P value of $<0.05$ was considered statistically significant.

\section{Establishment and validation of predicting nomogram model}

We hypothesized that patients who underwent primary tumor surgery and lived longer than the median CSS of the no-surgery group would benefit from surgery. Based on the above assumption, participants in the surgery group were classified into two types: a surgery-beneficial group (median CSS $>9$ months), and a surgery-non-beneficial group (median CSS $\leq 9$ months), according to the median CSS time of the 
no-surgery group (result from the matched cohort).

We used a logistic regression model to construct the predictive model for identifying candidates that would benefit from primary tumor surgery. Participants in the surgery group were used for analysis and split randomly into the training set ( $70 \%$ of participants) and validation set (30\% of participants).

According to the two categories above of surgery patients (surgery-beneficial vs. surgery-non-beneficial group), a multivariable logistic regression model was applied to predict surgery-benefitting patients. Variables included in the multivariable logistic regression models were selected a priori: age, gender, tumor location, histologic type, differentiation grade, and TNM stage. A nomogram was developed based on multivariate analysis on the training set to provide a quantitative tool to predict which $\mathrm{mEC}$ patients will benefit from primary site surgery.

The prediction performance of the nomogram was evaluated by the area under the receiver operating characteristic curve (AUC) on both the training and validation sets. A calibration plot was formulated to assess the calibration of the nomogram with the HosmerLemeshow goodness of fit test $(\mathrm{P}>0.05$ indicated insignificant deviance from the theoretical perfect calibration). Clinical usefulness and net benefit were estimated with decision curve analysis (DCA).

\section{Clinical utility of the nomogram}

The resultant predictive nomogram was employed in the surgery group to calculate the surgery benefit probability for each participant. A predictive benefit classification system was established to divide participants into two levels: (I) participant was classified as surgery benefit-candidate if the total prediction probability was $>0.5$; (II) participant with total prediction probability $\leq$ of 0.5 was classified as surgery no-benefit-candidate.

To test the nomogram's clinical application value, KaplanMeier analysis was conducted to test whether this predictive model would distinguish patients that could benefit from primary tumor surgery (surgery benefit-candidate $v s$. surgery no-benefit-candidate $v s$. no-surgery group).

\section{Results}

\section{Patients and baseline characteristics}

There was a total of 5,250 eligible patients included in this study. The selection process is shown in Figure 1. Of these mEC participants, 492 (9.4\%) underwent surgery to the primary tumor. The distributions of baseline characteristics for both study groups (surgery and nosurgery) are summarized and compared in Table 1, Tables S1,S2. As expected, there were obvious differences in age, gender, race, tumor location, histology, TNM stage, radiotherapy, chemotherapy, and surgery to distant sites between the two treatment groups, indicating that the two groups' baseline characteristics were not balanced.

The 1:1 PSM was utilized, and 472 matched pairs of $\mathrm{mEC}$ patients treated with or without primary site surgery were generated and enrolled in the following analysis. All baseline characteristics were well balanced after PSM (all $\mathrm{P}>0.05$ ), including age, gender, race, tumor location, histology, differentiation, TNM stage, radiotherapy, chemotherapy, and surgery to distant sites. Also, the standardized differences of baseline variables between study groups were all $<10 \%$ after matching (Figure S1). A detailed description of the treatment information of the matched cohort is provided in Figure S2.

\section{Correlation between surgery and survival in $m E C$}

The PSM cohort was included in the following analysis. In the Kaplan-Meier analysis, significant differences in survival outcome were observed when participants were stratified by primary site surgical intervention (Figure $2 A$ ). Those who received primary tumor surgery had longer median CSS (19 vs. 9 months; $\mathrm{P}<0.001)$ than those without surgery. The results revealed that the 1-year CSS rates were $64.0 \%$ in the surgery group and $40.3 \%$ in the no-surgery group. The 3 -year CSS rates were $40.9 \%$ in the surgery group and $10.1 \%$ in the no-surgery group. Multivariate Cox analysis further confirmed that primary tumor surgery was independently associated with improved CSS (HR 0.47; 95\% CI, 0.41-0.55, $\mathrm{P}<0.001$ ). Additionally, patients who underwent primary tumor surgery gained favorable CSS compared with those without primary tumor surgery across all baselinesubgroups with a relatively smaller HR (Figure 2B).

\section{Nomogram to identify benefit candidate for primary tumor surgery}

The analysis above showed a significantly longer survival in $\mathrm{mEC}$ patients with primary tumor surgery. In order to distinguish candidates that could benefit from surgery among mEC patients, we hypothesized that patients who 
Table 1 Characteristics for study population by study groups before and after PSM

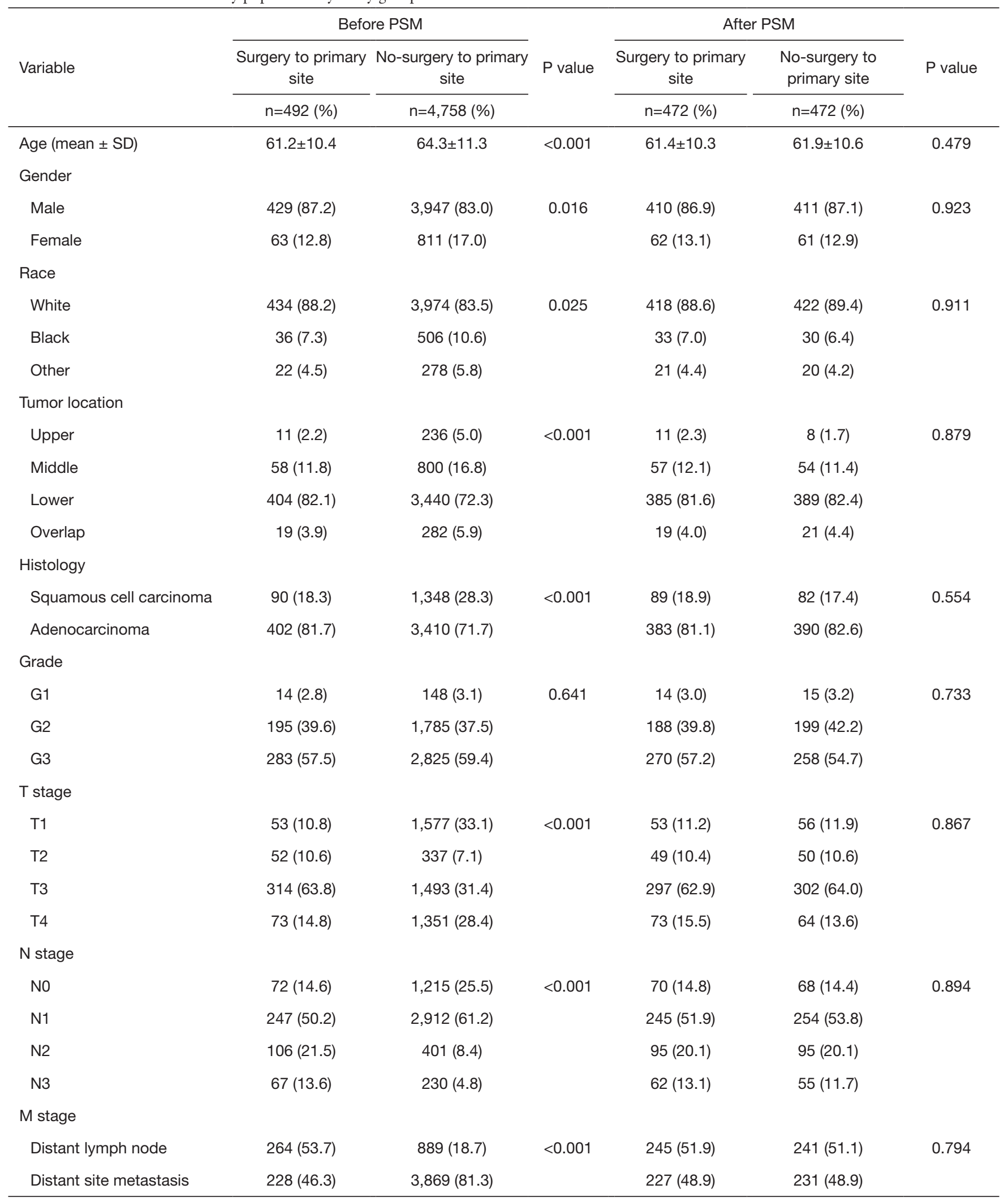

Table 1 (continued) 
Table 1 (continued)

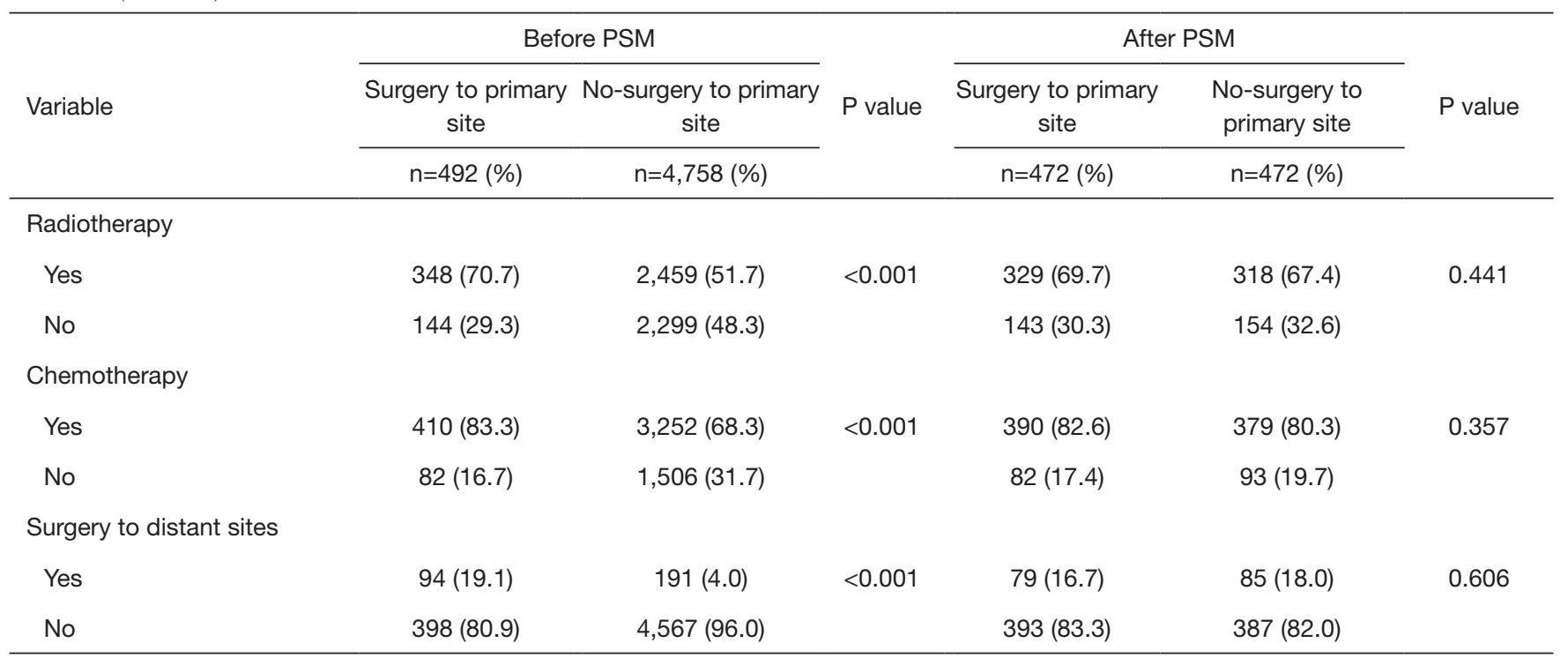

PSM, propensity-score matching; SD, standard deviation.

underwent surgery and lived longer than the median CSS of the no-surgery group ( 9 months) could benefit from surgery to the primary tumor. Among the surgery group, participants were divided into two categories: $69.3 \%$ [327] of patients survived $>9$ months and were allocated to the surgery-beneficial group, and those who had shorter CSS times were classified into the surgery-non-beneficial group.

Clinical baseline factors that can be accessed preoperatively were included for the surgery benefit nomogram, including age, gender, tumor position, differentiation grade, histology, and TNM stage. A prediction nomogram was developed based on the multivariable logistic analysis to predict the operable $\mathrm{mEC}$ patients who would benefit from primary site surgery in the training set. The prediction nomogram for surgery benefit candidate is presented in Figure 3.

The prediction nomogram had a good discrimination capacity for discerning the benefit-candidate from nobenefit-candidate in the training set (AUC $=0.722,95 \%$ CI: $0.663-0.781$ ) and the validation set (AUC $=0.701$, 95\% CI: 0.610-0.792) (Figure 4A,B). The calibration curves demonstrated good agreement between actual observation and prediction by nomogram in the training and the validation sets (both $\mathrm{P}>0.05$ ) (Figure $4 C, D$ ). DCA showed that using the nomogram to predict surgery benefit probability provided greater net benefit than either the "treat all with surgery" or "treat none with surgery" strategies for operable mEC patients, which indicated the clinical usefulness of the nomogram (Figure S3).

\section{Clinical utility of prediction nomogram}

The likelihood of surgery benefit of the individual mEC patients with operable performance status can be easily calculated by adding up each variable's corresponding prediction probability in the nomogram. A classification was developed according to the nomogram: the patient would be classed as a surgery benefit-candidate when the total prediction probability was beyond the cut-off point of 0.5 ; otherwise, the patient would be classed as a surgery nobenefit candidate. A schematic illustrating the application of the nomogram is shown in Figure 5. We then validated the distinguishability of the model in the validation set.

The nomogram's good discrimination capacity was demonstrated with the AUC, calibration curve, and DCA analysis above. We then tested the prediction of nomogram's clinical distinguishability by comparing the benefitcandidate, no-benefit candidate, and no-surgery groups. The Kaplan-Meier analysis showed that survival prognosis in the different groups was accurately differentiated in both the training and validation sets (Figure 6). In the validation set, the benefit-candidate group showed significantly better CSS than the no-benefit-candidate group (HR $=0.30,95 \%$ CI: $0.12-0.73, \mathrm{P}<0.001)$ and no-surgery group (HR $=0.51,95 \%$ CI: $0.42-0.62, \mathrm{P}<0.001)$, suggesting that the nomogram could sufficiently identify operable mEC 

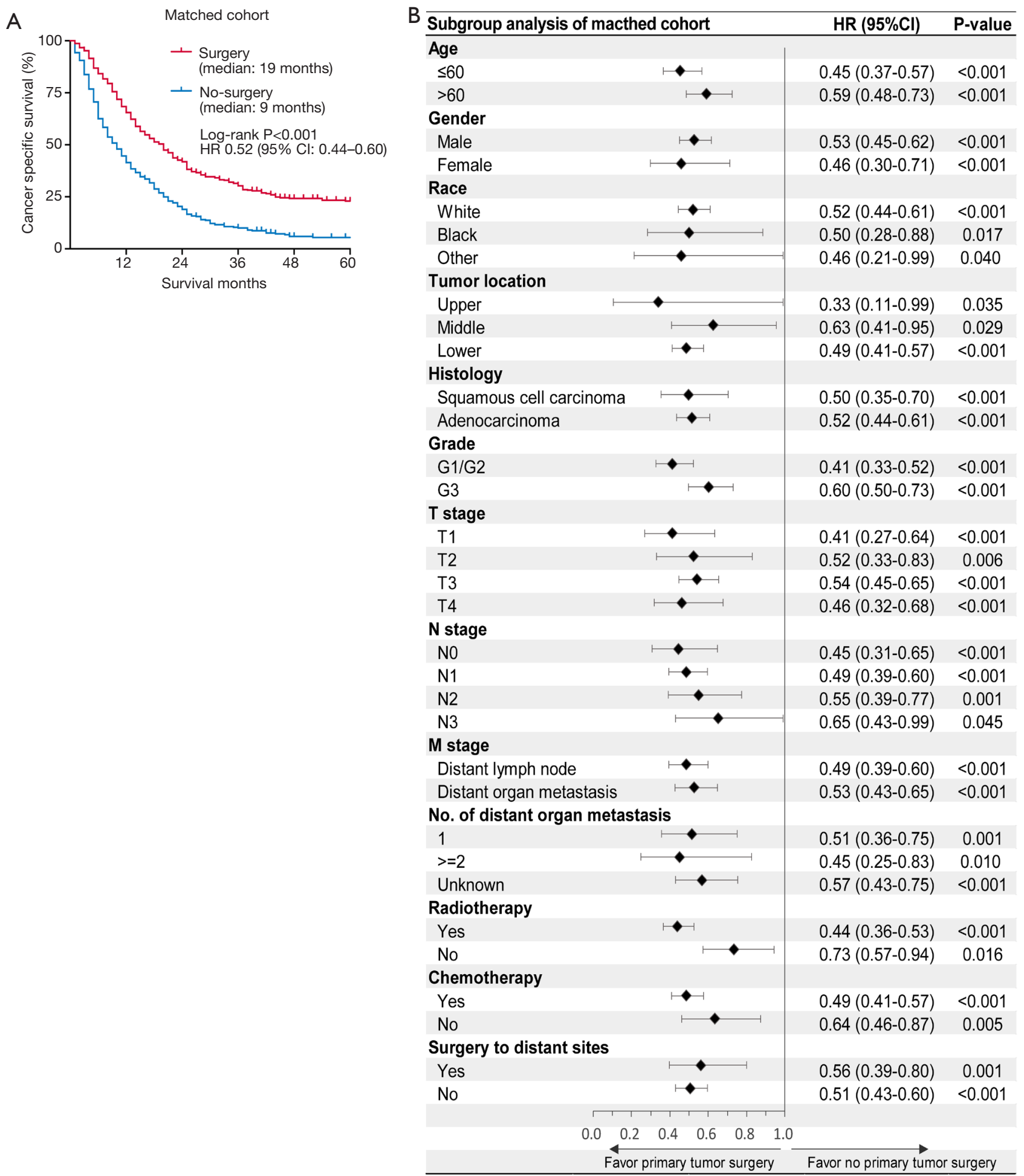

Figure 2 Comparison of cancer-specific survival between surgery to primary tumor vs. no surgery to primary tumor. (A) Plots of KaplanMeier estimates of cancer-specific survival of metastatic esophageal cancer patients with and without primary tumor surgery in the matched cohort. (B) Hazard ratios of cancer-specific survival for those who underwent primary tumor surgery, compared with those who did not undergo primary tumor surgery, by subgroups. [(B) Diamonds represent effect size (hazard ratio (HR)], calculated separately by primary tumor surgery $v s$. no primary tumor surgery in different subgroups; horizontal lines (error bars) indicate $95 \%$ confidence intervals (CIs). 


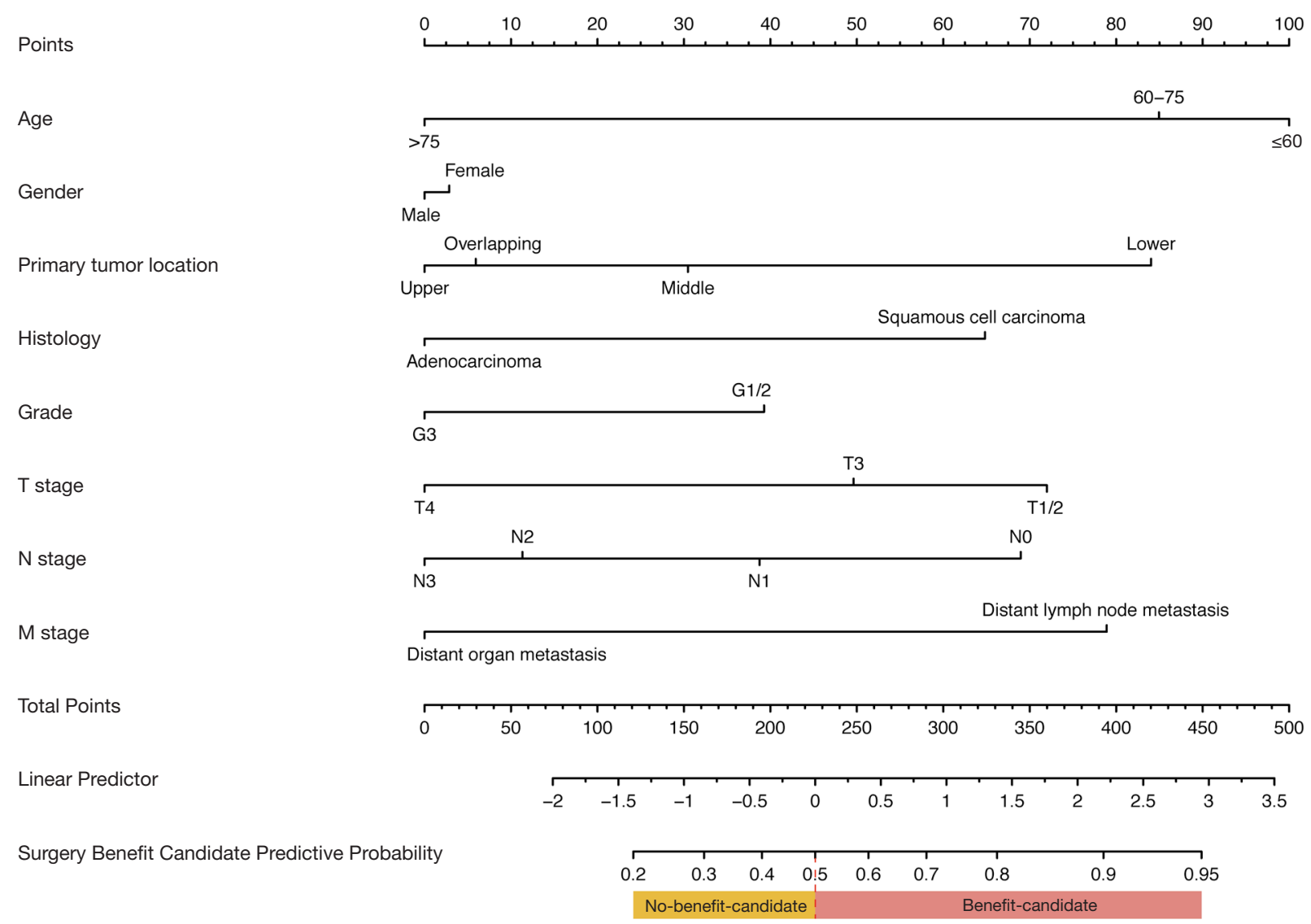

Figure 3 Prediction nomogram to predict candidate for benefit from primary tumor surgery in patients with metastatic esophageal cancer. The probability of each variable can be converted into scores according to the first scale "Points" at the top of the nomogram. After adding up the corresponding prediction probability at the bottom of the nomogram, the likelihood of surgery benefit of the individual patient can be calculated. The cut-off point of the nomogram is 0.5 . The patient would be classified as benefit-candidate when the total prediction probability is beyond the cut-off point.

patients who would benefit from primary tumor surgery. Moreover, there was no significant difference between the no-benefit-candidate group and no-surgery group ( $\mathrm{HR}=1.57,95 \%$ CI: $0.83-2.98, \mathrm{P}=0.070)$, indicating that the nomogram was able to identify some operable mEC patients who would not benefit from primary tumor surgery and for whom planning an appropriate non-surgical treatment strategy was required.

\section{Discussion}

This study was the first population-based analysis that assessed the role of a predictive model in identifying candidates that would benefit from primary tumor surgery in mEC. In this study, the beneficial effect of the survival outcome observed from the population database highlighted the potential of primary tumor surgery in the management of mEC. A prediction nomogram was developed to estimate candidate benefit for surgery to the primary tumor, which successfully stratified mEC participants according to their surgery benefit potential. The predictive factors included in the nomogram were conveniently available in clinical practice, and the validation of the prediction nomogram using different analyses demonstrated its positive performance and utility.

Systemic therapy, palliative/best supportive care, and sometimes clinical trials (e.g., immunotherapy) are preferred for mEC patients, while local treatment (e.g., surgery to the primary tumor) is not usually recommended. Notably, substantial heterogeneity exists among patients with mEC 

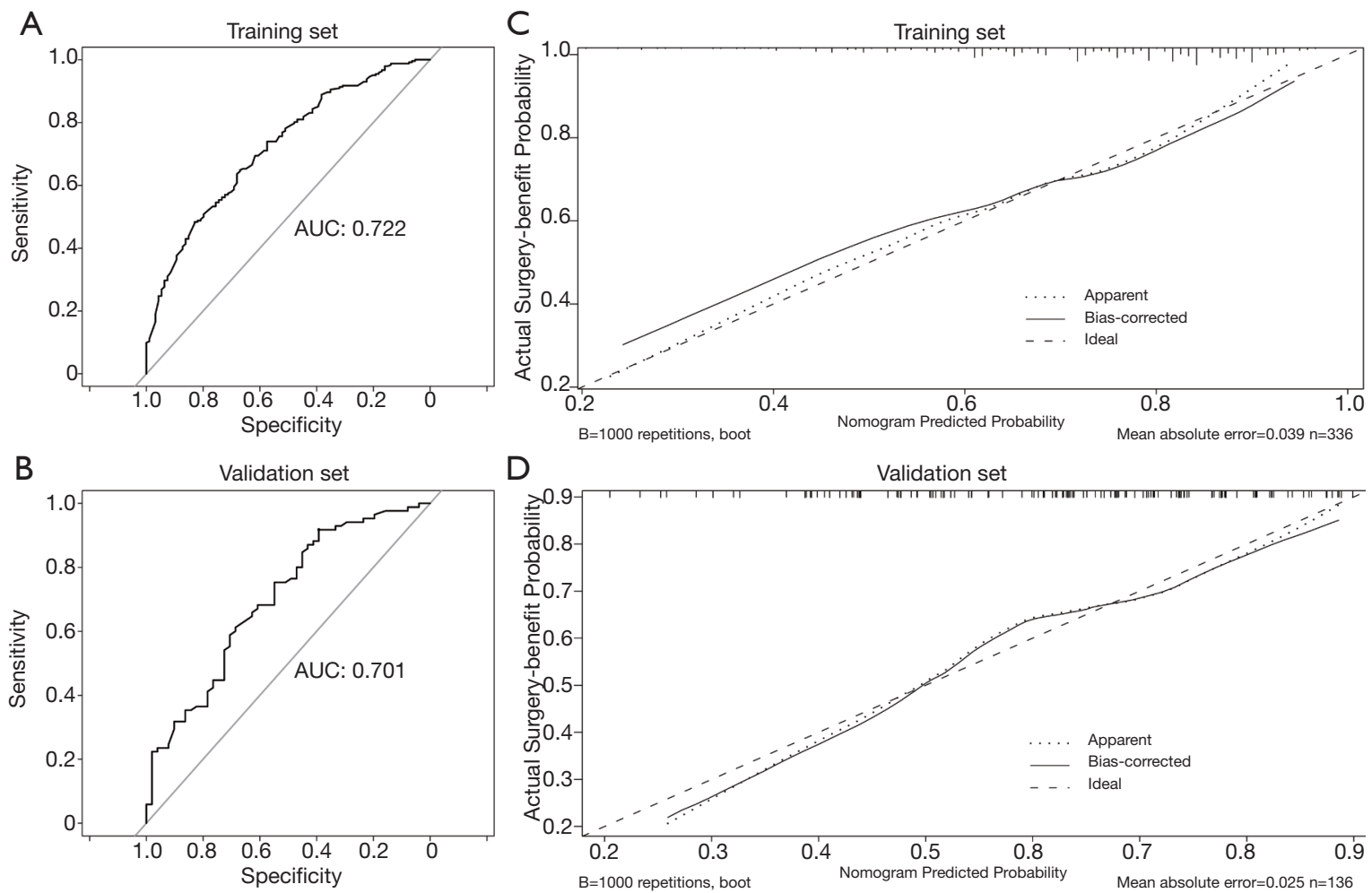

Figure 4 Validity of the predictive performance of the nomogram with ROC and calibration curves. ROC curves of the nomogram in the training (A) and validation (B) sets. Calibration curves of the nomogram in the training (C) and validation (D) sets. ROC, receiver operating characteristic.

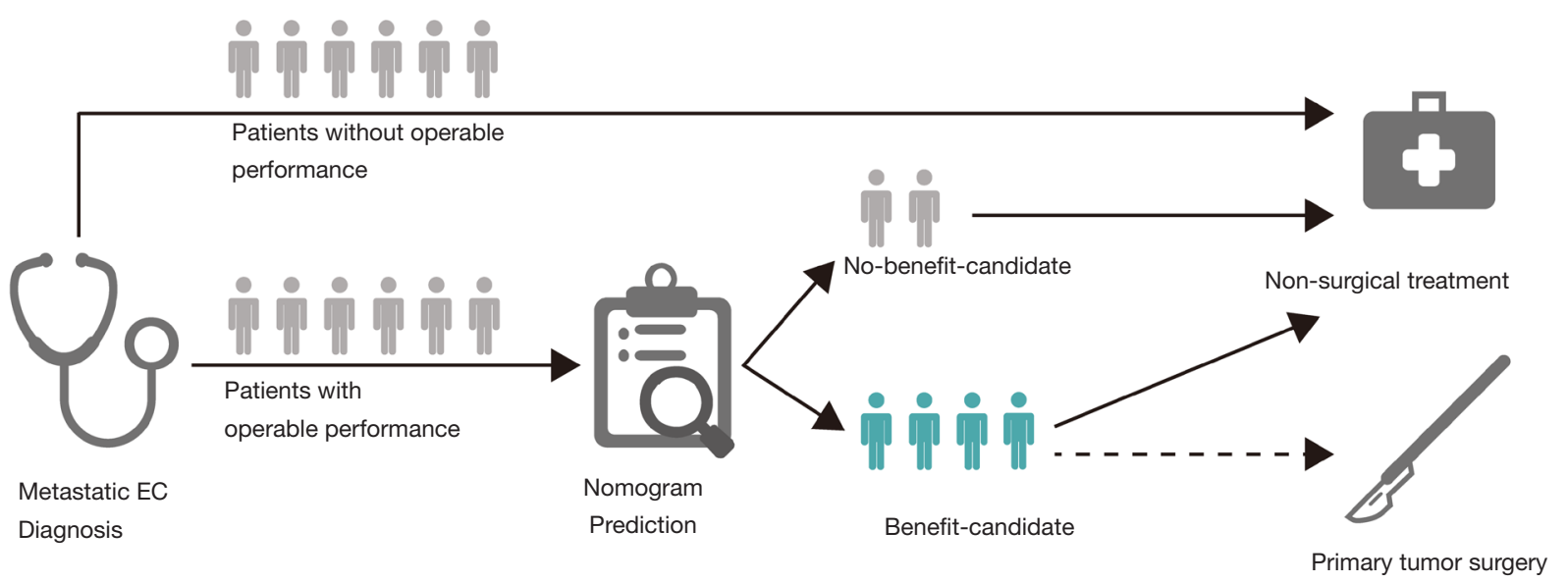

Figure 5 A schematic depicting the application of the nomogram. In this analysis, a prediction nomogram was developed to identify candidate for benefit from primary tumor surgery in metastatic stage esophageal cancer, and provide more treatment options to these patients. 
A

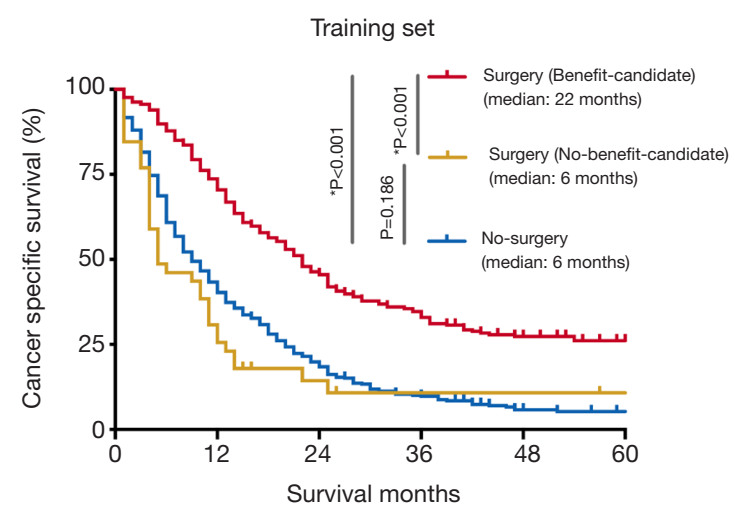

B

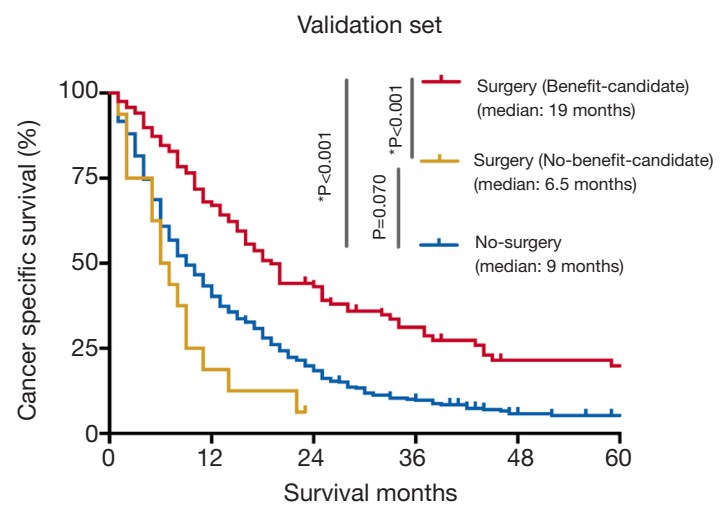

Figure 6 Kaplan-Meier curves of survival for metastatic esophageal cancer patients in different benefit classification according to the nomogram (benefit-candidate and no-benefit candidate groups) and no-surgery group.

regarding clinicopathological information, such as age, tumor location, and tumor stage. Thus, the prognosis of $\mathrm{mEC}$ varies across different cases, and the treatment management remains in need of further exploration. The inclusion of surgery into multimodality treatment has been reproducibly associated with improved prognosis in patients with locally advanced stage EC $(13,14)$; however, the role of surgical intervention has remained unclear for $\mathrm{mEC}$. In fact, resection of the primary tumor is associated with improved survival prognosis in many metastatic stage cancers (15-18), which might be due to the great reduction of tumor burden by primary tumor resection, which subsequently reverses the detrimental effects of tumor progression on the immune response (19).

Previous studies have questioned the value of primary tumor surgery and found that surgery did not improve survival in $\mathrm{mEC}$ (20). Regarding the postoperative morbidity and mortality, no significant difference was found between mEC and locally advanced EC (21). However, more recent studies have revealed the benefit of primary tumor surgery in $\mathrm{mEC}$ patients $(6,7,9)$. Other related studies have also revealed that primary tumor resection could prolong survival in mEC patients after multimodality therapy compared with single-modality treatment and best supportive care (10,22-24). Certain characteristics have been linked to improved patient survival, for example, young age, fewer comorbidities, less nodal invasion, and limited metastasis. These findings indicated the role of local control in improving patients' prognosis. Also, primary tumor resection for judiciously selected $\mathrm{mEC}$ patients could prevent tumor-related complications such as obstruction, bleeding, and perforation, thus reducing the potential risk of severe tumor-related complications.

Our results were in line with other studies in that patient who underwent primary tumor surgery had prolonged survival outcomes. The median CSS of patients receiving primary tumor surgery was 19 months, which was almost 10 months longer than those without surgery. The analysis data was based on a population database with the intention that the results would reflect mEC patients' outcomes in the real world. Of note, the results showed that some participants treated with surgery failed to reach the median CSS time (9 months) of those without surgery. In other words, not all operable patients could benefit from the surgery in terms of survival, indicating the insufficiency of the surgery recommendation. Therefore, we came up with a novel process to establish a prediction model to optimize candidate selection to those who would gain prolonged survival outcomes from primary tumor surgery. The median CSS of the surgery and no-surgery groups were 19 and 9 months, respectively. We divided the surgery participants into two subgroups: we assumed that primary tumor surgery participants who lived $>9$ months could benefit from the surgery (surgery-beneficial group); while those who failed to survive $>9$ months were considered as the surgery-nonbeneficial group. The surgery-beneficial group $v s$. surgerynon-beneficial group was then analyzed using a multivariate logistic model, including the preoperative baseline factors, and a prediction nomogram developed accordingly. Using this prediction nomogram, $\mathrm{mEC}$ patients who were suitable for surgery were identified with the individualized prediction of the surgery benefit potential. Our prediction 
nomogram was an important supplementary tool to assist in identifying optimal mEC patients for surgical intervention at diagnosis. Clinicians can use the prediction nomogram to estimate an individual patient's potential for surgery benefit. According to this prediction nomogram, patients classified as surgery benefit-candidates are more likely to gain benefit from primary tumor surgery, and in this case, surgical therapy might be recommended as a treatment option in addition to non-surgical therapy. For patients classified as surgery no-benefit-candidates, a non-surgical treatment strategy would be a reasonable recommendation. We believe that clinicians are better informed by calculating the benefit estimate for each patient and making decisions based on multidisciplinary team collaboration.

The observation of improved survival with surgical intervention is possibly attributed to selection bias for patients with favorable individual factors (e.g., younger age, better performance status, lower-tumor burden). However, surgery may have a therapeutic impact that is obscured by the selection bias, and that selection bias only represents a part rather than the total of the improved prognosis observed in mEC patients treated with surgery. In our predictive nomogram, younger age and tumor location were the top two strongest predictors of benefit from primary tumor surgery in $\mathrm{mEC}$ patients, suggesting that certain individual conditions are key to selecting surgical treatment for mEC patients. As is described in previous studies, younger patients, lower nodal status, and limited metastasis were associated with better outcomes for $\mathrm{mEC}$ patients with primary tumor surgery, which might be due to the relatively reduced surgical difficulty and associated risks (10). These factors are known to favorably influence prognosis, reinforcing the concept that patients with an expectation of longer survival and better individual conditions are potentially offered higher risk therapy, such as surgical intervention. Therefore, it might be reasonable to provide primary tumor surgery as a treatment option for certain $\mathrm{mEC}$ patients with potential surgery benefits. However, up to now, there has been no definite selection criteria of mEC patients who would potentially benefit from surgical therapy. The aggregation of multiple clinical factors might have a stronger prediction benefit than any simple single indicator. An individualized prediction would be an ideal assistant-selection tool for this situation. Thus, this exploratory study developed an individualized prediction model to identify surgery benefit candidates to contribute to future research and provide more prognostic information on clinicians and patients' treatment decisions.
There were several limitations to this study. First, this study was a retrospective analysis with inherent biases as with any observational studies. Second, the SEER database did not provide information regarding performance status and comorbidity that might have led to a selection bias for treatment choice; however, our novel predictive nomogram was developed based on a surgery population who were evaluated as operable performance preoperatively, which had already specified the condition to use the nomogram. Third, some detailed information could not be acquired from the SEER database, such as a systematic therapy regimen, metastatic site, number of metastatic tumors, resection type (complete or incomplete), and treatment before and after surgery might represent a limitation of the current study. We could not answer whether the primary tumor resection enhanced survival benefit more if performed before or after the systemic treatment. Finally, although the prediction nomogram was developed using a population cohort and validated in a split subgroup of participants, the nomogram's external validation needs to be performed in future studies with large sample sizes.

In summary, only specific $\mathrm{mEC}$ patients are likely to benefit from primary tumor surgery, and we established and validated a predictive model to identify benefit candidates for primary tumor surgery. This predictive model incurs no further cost. Its use could help select optimal candidates for primary tumor surgery among mEC patients and, therefore, provide an additional effective treatment option to them, with potential prognosis benefit. The predictive model merits further prospective validation and future improvement predictive model is warranted.

\section{Acknowledgments}

Funding: This study was funded by grants from three years of clinical innovation action plan (16CR1035B) and Shanghai Municipal Education Commission-Gaofeng Clinical Medicine Grant Support.

\section{Footnote}

Reporting Checklist: The authors have completed the TRIPOD reporting checklist. Available at http://dx.doi. org/10.21037/jtd-20-2347

Conflicts of Interest: All authors have completed the ICMJE uniform disclosure form (available at http://dx.doi. org/10.21037/jtd-20-2347). The authors have no conflicts 
of interest to declare.

Ethical Statement: The authors are accountable for all aspects of the work in ensuring that questions related to the accuracy or integrity of any part of the work are appropriately investigated and resolved. The study was conducted in accordance with the Declaration of Helsinki (as revised in 2013). This study was based on the publicly available data from the Surveillance, Epidemiology, and End Results (SEER) database and we obtained the permission to access the research data. In this study, informed consent was not required for patients, and only de-identified and publicly available data were used. As all the data were anonymous, it was deemed exempt by the Institutional Review Board of the Shanghai Chest Hospital.

Open Access Statement: This is an Open Access article distributed in accordance with the Creative Commons Attribution-NonCommercial-NoDerivs 4.0 International License (CC BY-NC-ND 4.0), which permits the noncommercial replication and distribution of the article with the strict proviso that no changes or edits are made and the original work is properly cited (including links to both the formal publication through the relevant DOI and the license). See: https://creativecommons.org/licenses/by-nc-nd/4.0/.

\section{References}

1. Bray F, Ferlay J, Soerjomataram I, et al. Global cancer statistics 2018: GLOBOCAN estimates of incidence and mortality worldwide for 36 cancers in 185 countries. CA Cancer J Clin 2018;68:394-424.

2. Rustgi AK, El-Serag HB. Esophageal carcinoma. N Engl J Med 2014;371:2499-509.

3. Enzinger PC, Ilson DH, Kelsen DP. Chemotherapy in esophageal cancer. Semin Oncol 1999;26:12-20.

4. Ku GY. Systemic therapy for esophageal cancer: chemotherapy. Chin Clin Oncol 2017;6:49.

5. Enzinger PC, Burtness BA, Niedzwiecki D, et al. CALGB 80403 (Alliance)/E1206: A Randomized Phase II Study of Three Chemotherapy Regimens Plus Cetuximab in Metastatic Esophageal and Gastroesophageal Junction Cancers. J Clin Oncol 2016;34:2736-42.

6. Zhang R, Zou J, Li P, et al. Surgery to the primary tumor is associated with improved survival of patients with metastatic esophageal cancer: propensity score-matched analyses of a large retrospective cohort. Dis Esophagus 2020;33:doz051.
7. $\mathrm{Xu} \mathrm{J}, \mathrm{Lu} \mathrm{D}$, Zhang $\mathrm{L}$, et al. Palliative resection or radiation of primary tumor prolonged survival for metastatic esophageal cancer. Cancer Med 2019;8:7253-64.

8. Saddoughi SA, Reinersman JM, Zhukov YO, et al. Survival After Surgical Resection of Stage IV Esophageal Cancer. Ann Thorac Surg 2017;103:261-6.

9. Blank S, Lordick F, Dobritz M, et al. A reliable risk score for stage IV esophagogastric cancer. Eur J Surg Oncol 2013;39:823-30.

10. Wang J, Suri JS, Allen PK, et al. Factors Predictive of Improved Outcomes With Multimodality Local Therapy After Palliative Chemotherapy for Stage IV Esophageal Cancer. Am J Clin Oncol 2016;39:228-35.

11. McCaffrey DF, Ridgeway G, Morral AR. Propensity score estimation with boosted regression for evaluating causal effects in observational studies. Psychol Methods 2004;9:403-25.

12. Austin PC. Balance diagnostics for comparing the distribution of baseline covariates between treatment groups in propensity-score matched samples. Stat Med 2009;28:3083-107.

13. Yang H, Liu H, Chen Y, et al. Neoadjuvant Chemoradiotherapy Followed by Surgery Versus Surgery Alone for Locally Advanced Squamous Cell Carcinoma of the Esophagus (NEOCRTEC5010): A Phase III Multicenter, Randomized, Open-Label Clinical Trial. J Clin Oncol 2018;36:2796-803.

14. van Hagen P, Hulshof MC, van Lanschot JJ, et al. Preoperative chemoradiotherapy for esophageal or junctional cancer. N Engl J Med 2012;366:2074-84.

15. Ahmed S, Leis A, Fields A, et al. Survival impact of surgical resection of primary tumor in patients with stage IV colorectal cancer: results from a large population-based cohort study. Cancer 2014;120:683-91.

16. Musri FY, Mutlu H, Karaagac M, et al. Primary Tumor Resection and Survival in Patients with Stage IV Gastric Cancer. J Gastric Cancer 2016;16:78-84.

17. Yang CJ, Gu L, Shah SA, et al. Long-term outcomes of surgical resection for stage IV non-small-cell lung cancer: A national analysis. Lung Cancer 2018;115:75-83.

18. Mudgway R, Chavez de Paz Villanueva C, Lin AC, et al. The Impact of Primary Tumor Surgery on Survival in HER2 Positive Stage IV Breast Cancer Patients in the Current Era of Targeted Therapy. Ann Surg Oncol 2020;27:2711-20.

19. Danna EA, Sinha P, Gilbert M, et al. Surgical removal of primary tumor reverses tumor-induced immunosuppression despite the presence of metastatic 
disease. Cancer Res 2004;64:2205-11.

20. Tanaka T, Fujita H, Matono S, et al. Outcomes of multimodality therapy for stage IVB esophageal cancer with distant organ metastasis (M1-Org). Dis Esophagus 2010;23:646-51.

21. Schauer M, Stein H, Lordick F, et al. Results of a Multimodal Therapy in Patients with Stage IV Barrett's Adenocarcinoma. World J Surg 2008;32:2655-60.

22. Wu SG, Xie WH, Zhang ZQ, et al. Surgery Combined with Radiotherapy Improved Survival in Metastatic Esophageal Cancer in a Surveillance Epidemiology

Cite this article as: Liu Z, Zhang X, Li B, Jiang H, Yang Y, Hua R, Sun Y, Li Z. A population-based predictive model predicting candidate for primary tumor surgery in patients with metastatic esophageal cancer. J Thorac Dis 2021;13(2):870-882. doi: $10.21037 /$ jtd-20-2347 and End Results Population-based Study. Sci Rep 2016;6:28280.

23. Chao YK, Wu YC, Liu YH, et al. Distant Nodal Metastases From Intrathoracic Esophageal Squamous Cell Carcinoma: Characteristics of Long-Term Survivors After Chemoradiotherapy. J Surg Oncol 2010;102:158-62.

24. Mudan SS, Giakoustidis A, Giakoustidis D, et al. Synchronous oesophagectomy and hepatic resection for metastatic oesophageal cancer: report of a case. Hippokratia 2010;14:291-3. 


\section{Supplementary}

Table S1 Surgical procedure of patients who underwent primary tumor surgery

\begin{tabular}{|c|c|c|c|c|c|c|}
\hline & & \multicolumn{3}{|c|}{ Before PSM } & \multicolumn{2}{|l|}{ After PSM } \\
\hline \multicolumn{7}{|c|}{ Resection of primary site tumor } \\
\hline Total esophagectomy & & \multicolumn{3}{|c|}{$397(80.7)$} & \multicolumn{2}{|l|}{$379(80.3)$} \\
\hline Partial esophagecton & & \multicolumn{3}{|c|}{$45(9.1)$} & \multicolumn{2}{|l|}{$43(9.1)$} \\
\hline Number of retrieved LN & (IQR) & \multicolumn{3}{|c|}{$12[6-19]$} & \multicolumn{2}{|l|}{$12[6-19]$} \\
\hline \multicolumn{7}{|c|}{$\begin{array}{l}\text { a, primary tumor resection was performed, but did not specify the procedure. PSM, propensity-score matching; NOS, not otherwise } \\
\text { specific; LN, lymph node; IQR, interquartile range. }\end{array}$} \\
\hline & $\mathrm{n}=492(\%)$ & $\mathrm{n}=4758(\%)$ & P-value & $n=472(\%)$ & $n=472(\%)$ & P-value \\
\hline Distant lymph nodes & $264(53.7)$ & $889(18.7)$ & $<0.001$ & $245(51.9)$ & $241(51.1)$ & 0.982 \\
\hline \multicolumn{7}{|c|}{ Number of metastatic organs } \\
\hline 1 & 75 (15.2) & $1250(26.3)$ & & $75(15.9)$ & 77 (16.3) & \\
\hline 2 & $27(5.5)$ & $498(10.5)$ & & $27(5.7)$ & $31(6.6)$ & \\
\hline$>=3$ & $8(1.6)$ & $122(2.6)$ & & $8(1.7)$ & $7(1.5)$ & \\
\hline
\end{tabular}

\footnotetext{
a , distant organ metastasis was reported, but without detailed information. PSM, propensity-score matching.
} 


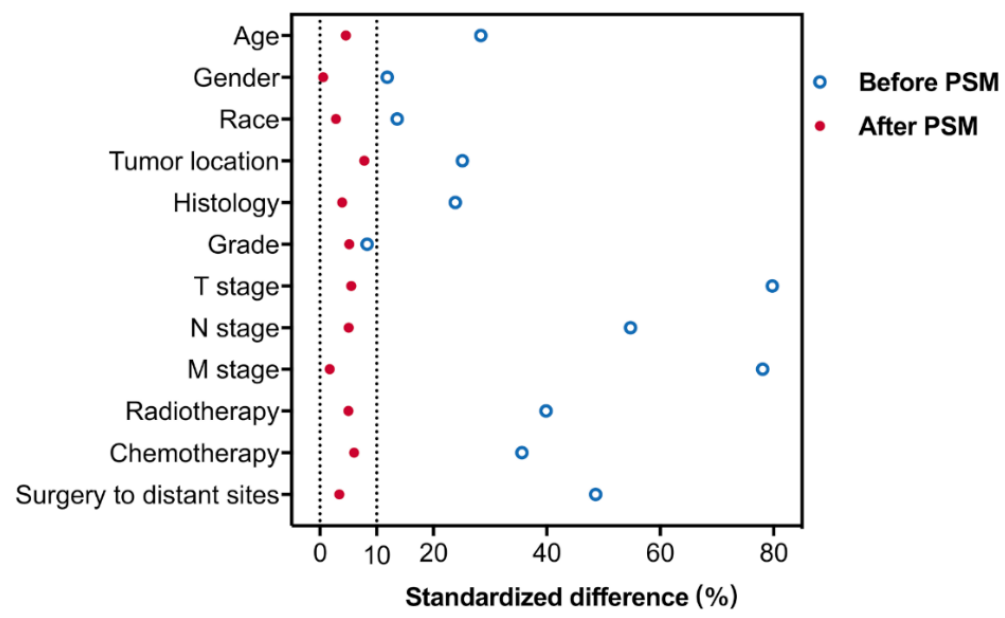

Figure S1 Standardized differences of baseline variables between patients with and without primary tumor surgery before and after PSM. PSM, propensity score matching.

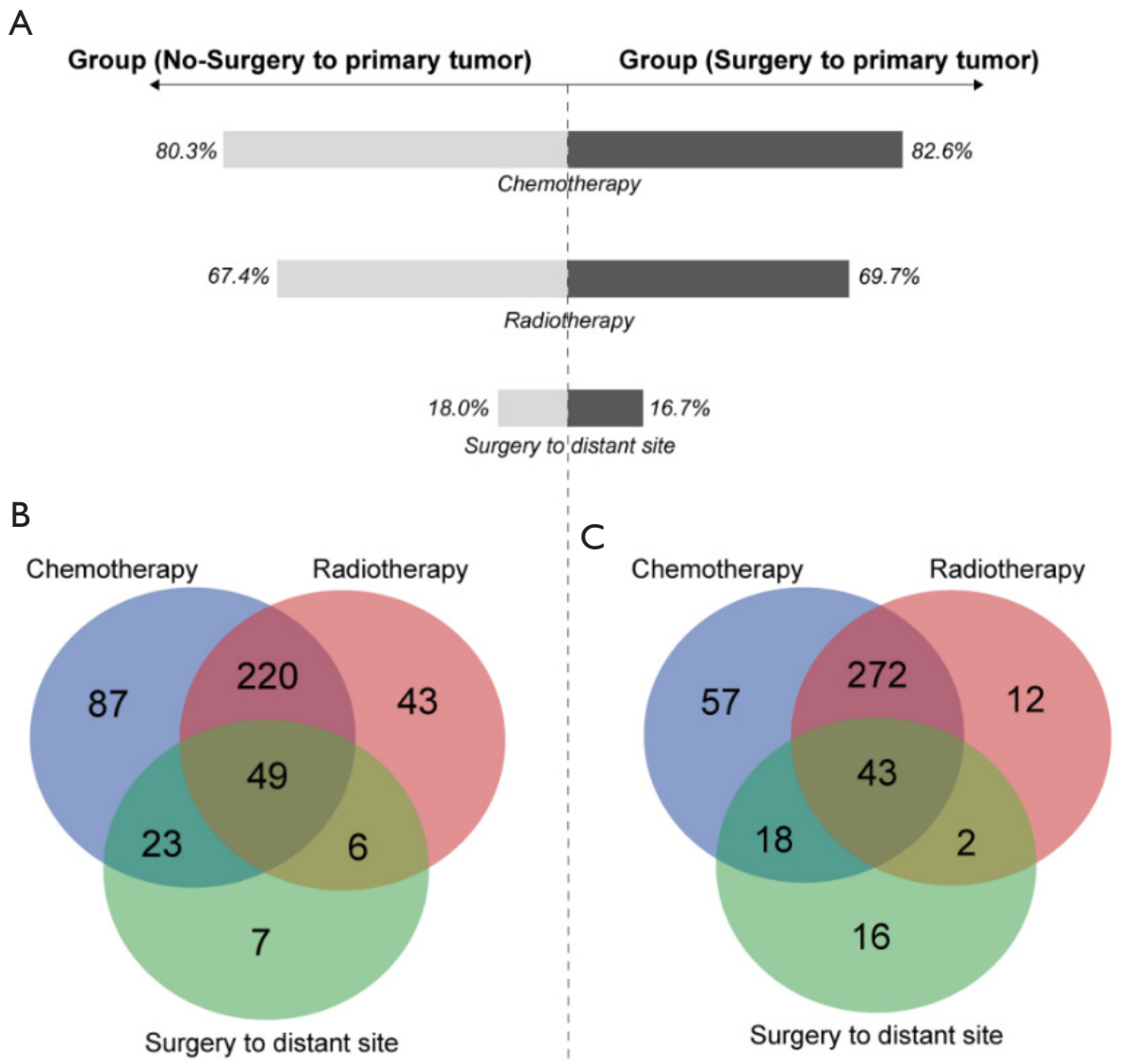

Figure S2 Treatment modalities in patients with and without primary tumor surgery in the matched cohort. (A) Bar chart showing treatment between the two groups (surgery to primary tumor $v$ s. no-surgery to primary tumor). (B) Venn diagram showing treatment in the group of no-surgery to primary tumor. (C) Venn diagram showing treatment in the group of surgery to primary tumor. 


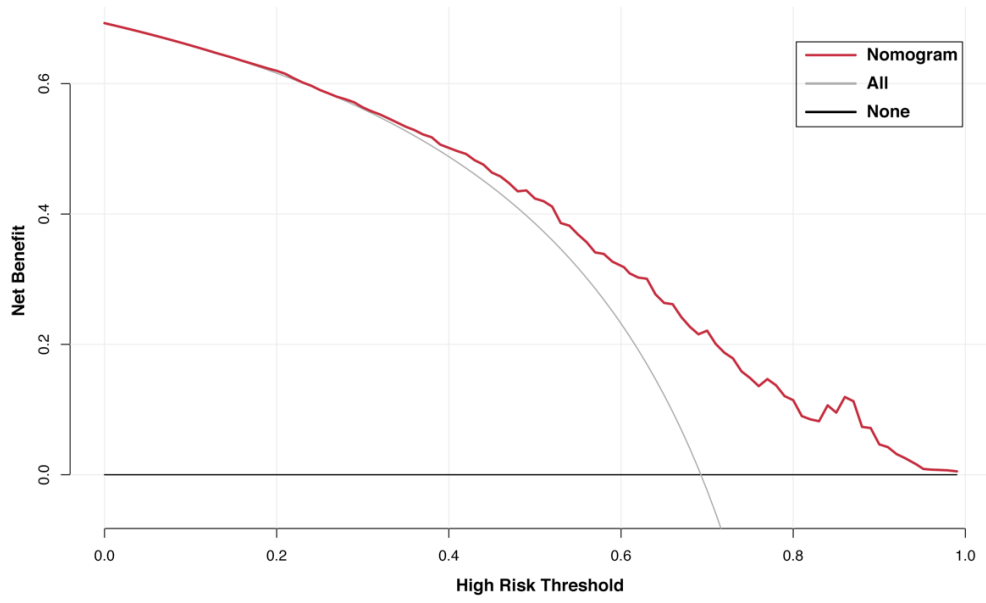

Figure S3 Decision curve analysis for the prediction nomogram. The $\mathrm{X}$-axis represents the threshold probability. The Y-axis measures the net benefit. The red line represents the prediction nomogram. The grey and black line represent the hypothesis that all patients were benefit candidates or no-benefit-candidates, respectively. 